\title{
Use of national databases: interpreting treatment outcomes using real-world data
}

\author{
Melanie Subramanian, Benjamin D. Kozower \\ Division of Cardiothoracic Surgery, Washington University School of Medicine, St. Louis, MO, USA \\ Correspondence to: Benjamin D. Kozower, MD, MPH. S. Euclid Avenue, Campus Bos 8234, St. Louis, MO 63110, USA. Email: kozowerb@wustl.edu. \\ Provenance: This is an invited article commissioned by the Section Editor Gang Shen (The Second Affiliated Hospital Zhejiang University School of \\ Medicine, Hangzhou, China). \\ Response to: Cao C, Tian DH, Fu B, et al. The problem with sublobar resections. J Thorac Dis 2018;10:S3224-6.
}

Submitted Oct 18, 2018. Accepted for publication Nov 23, 2018.

doi: $10.21037 /$ jtd.2018.11.129

View this article at: http://dx.doi.org/10.21037/jtd.2018.11.129

In our paper "Long-Term Results for Clinical Stage IA Lung Cancer: Comparing Lobectomy and Sublobar Resection", we used an enhanced dataset from the National Cancer Database (NCDB) to compare 5-year overall survival (OS) and disease recurrence in propensity matched patients undergoing lobectomy or sublobar resection for clinical stage IA non-small cell lung cancer (NSCLC) (1). We found that 5 -year OS was similar between cohorts. However, those undergoing sublobar resection were identified to have a $39 \%$ increased risk of cancer recurrence.

We appreciate the commentary brought by $\mathrm{Cao}$ and colleagues. Principally, they highlight several limitations associated with the use of a large national database (2). The extent of analysis that can be performed using a large database is limited by the granularity of the data and the sample size afforded after stringent inclusion and exclusion criteria are applied. We fully acknowledge that in the ideal situation, we would have sufficient sample size to differentiate between segmentectomy and wedge resection. This is due to existing literature suggesting that segmentectomy and wedge are associated with different short and long-term survival and oncologic outcomes (3). However, given the sampling strategy used by the American College of Surgeons Special Study that was utilized by our group, the number of patients who underwent segmentectomy was fairly limited. In the special study, up to 10 patients who underwent surgical resection for stage I-III NSCLC with complete follow-up data were selected from each participating commission on cancer site. Thus, our sample size (which only included surgically resected clinical stage IA patients) was considerably smaller than what is normally available in the NCDB. Our sample size was further reduced after propensity matching was applied to ensure that we utilized a study cohort where there is greater equipoise in treatment selection. Another important limitation is the level of granularity of the dataset. While 15 comorbidity variables and recurrence data were abstracted from medical records, these did not include pulmonary function tests or measures of overall functional status. These are important considerations when determining extent of resection and we recognize them in our manuscript.

However, use of national databases offer the opportunity to perform similar studies and reflect real world outcomes. They are important and different from randomized controlled trials (RCTs) and single-institution studies in that they provide generalizability. The NCDB includes approximately $70 \%$ of incident cancers (4). Not only do they capture a diverse patient population representative of NSCLC in the United States, they include patients from hospitals of varying volume and specialization. While Cao and colleagues note that advances in histologic subtyping and examination of spread through air spaces (STAS) can provide important adjuncts to determination of extent of resection, there remains insufficient evidence that these techniques have been adopted routinely in the majority of practices (2).

There is ultimately a disconnect between what ideally should happen, and what is happening in the real world. Ideally, most surgeons would adhere to quality 
measures such as sufficient lymph node sampling and ensuring sufficient margin distance in wedge resection by confirmation with intra-operative frozen section. As shown in the data, this is not happening. Patients undergoing sublobar resection in our study cohort had a median lymph node sampling count of just one node, and they had a higher frequency of positive margins (1). Performing (RCTs) to address this question will not solve this issue-they incorporate best practices into rigid study protocols, and thus all results and conclusions from these studies must be interpreted in this context.

In addition to RCTs or those that are assessing novel technologies and their application to treatment allocation, studies that have an emphasis on using generalizable data also deserve consideration. Ideally, surgeons who are performing lung cancer operations are incorporating the latest evidence-based practices and are adhering to established quality measures when performing either lobectomy or sublobar resection. However, as we have demonstrated, there is a clear disconnect between what should happen and what is happening in the real world. It is important for us to take the real-world consequences of treatment decisions into consideration. We observed that patients who were undergoing sublobar resection were often not meeting the same quality measures as those undergoing lobectomies. Given our findings in this context, we would still advocate for an anatomic operation for those who have the physical and pulmonary capacity to tolerate such a procedure.

Cite this article as: Subramanian M, Kozower BD. Use of national databases: interpreting treatment outcomes using realworld data. J Thorac Dis 2018;10(12):E828-E829. doi: 10.21037/ jtd.2018.11.129

\section{Acknowledgments}

Funding: Funding support was provided by the Patient Centered Outcomes Research Institute (R-APD-1306-00727). Dr. B Kozower, corresponding author, is the grant recipient. M Subramanian received funding support from the T32 NIH Cardiothoracic Training Grant (Grant Number: 5T32HL007776023).

\section{Footnote}

Conflicts of Interest: The authors have no conflicts of interest to declare.

\section{References}

1. Subramanian M, McMurry T, Meyers BF, et al. LongTerm Results for Clinical Stage IA Lung Cancer: Comparing Lobectomy and Sublobar Resection. Ann Thorac Surg 2018;106:375-81.

2. Cao C, Tian DH, Fu B, et al. The problem with sublobar resections. J Thorac Dis 2018;10:S3224-6 .

3. Smith CB, Swanson SJ, Mhango G, et al. Survival after segmentectomy and wedge resection in stage I non-smallcell lung cancer. J Thorac Oncol 2013;8:73-8.

4. Surgeons ACo. CoC Quality of Care Measures. 2014. Available online: https://www.facs.org/quality-programs/ cancer/ncdb/qualitymeasures 